\title{
Experimental investigation on the impact of connate water salinity on dispersion coefficient in consolidated rocks cores during Enhanced Gas Recovery by $\mathrm{CO}_{2}$ injection

\author{
M.K. Abba ${ }^{1}$, A. Al-Otaibi ${ }^{1}$, A.J. Abbas ${ }^{1}$, Mukhtar Abdulkadir ${ }^{2}$ \\ Journal of Natural Gas Science and Engineering \\ 1 The University of Salford Manchester UK \\ 2 Federal University of Technology, Minna, Nigeria
}

\begin{abstract}
Connate water salinity is a vital property of the reservoir and its influence on the displacement efficiency cannot be overemphasised. Despite the numerous analytical literatures on the dispersion behaviour of $\mathrm{CO}_{2}$ in $\mathrm{CH}_{4}$ at different parametric conditions, studies have so far been limited to systematic effects of the process while parameters such as connate water salinity of the reservoir has not been given much attention and this could redefine the $\mathrm{CO}_{2}-\mathrm{CH}_{4}$ interactions in the reservoir. This study aims to experimentally determine the effect of connate water salinity on the dispersion coefficient in consolidated porous media under reservoir conditions. A laboratory core flooding experiment depicting the detailed process of the $\mathrm{CO}_{2}-\mathrm{CH}_{4}$ displacement using Grey Berea sandstone core sample at a temperature of $50^{\circ} \mathrm{C}$ and at a pressure of $1300 \mathrm{psig}$ was carried out to determine the optimum injection rate, from $0.2-0.5 \mathrm{ml} / \mathrm{min}$, for the experimentation based on dispersion coefficients and methane recovery in the horizontal orientation. This was established to be $0.3 \mathrm{ml} / \mathrm{min}$. At the same conditions, the effects of connate water saturation of $10 \%$ and a salinity of 0 (distilled water), 5 , and $10 \%$ wt. with a $\mathrm{CO}_{2}$ injection rate of $0.3 \mathrm{ml} / \mathrm{min}$ on the dispersion coefficients was investigated. The results from the core flooding process indicated that the dispersion coefficient decreases with increasing salinity, hence the higher the density of the immobile phase (connate water) the lower the dispersion of $\mathrm{CO}_{2}$ into $\mathrm{CH}_{4}$. This is a significant finding given that the inclusion of the connate water and its salinity have an effect on the mixing of the gases in the core sample and should be given importance and included during simulation studies for field scale applications of Enhanced Gas Recovery (EGR). This is the first experimental investigation into the relationship between the connate water salinity and the dispersion coefficient in consolidated porous media.

Keywords: Enhanced Gas Recovery; Dispersion Coefficient; Connate water Salinity; CO2 sequestration
\end{abstract}

\section{Introduction}

As natural gas continues to gain widespread usage as a source of cleaner and efficient fossil fuel, and greenhouse gas emission is attracting environmental consequences, the need for a viable method to enhance and curtail these phenomena, respectively, is paramount (Al-abri et al., 2009; Al-Abri et al., 2012; Al-Abri, 2011; Benson et al., 2005; Benson and Cole, 2008; Oldenburg and Benson, 2002). The technique of injecting $\mathrm{CO}_{2}$ into deep saline aquifers and oil and gas reservoirs have the potential for alternative methods for reducing $\mathrm{CO}_{2}$ emissions (Vilcáez, 2015). Studies (Allen et al., 2017; Bennaceur, 2013; Riis and Halland, 2014; Sanguinito et al., 2018; Sminchak et al., 2017) have shown that deep saline aquifers have the advantage of more storage capacity as a result of different storage mechanisms over oil and gas reservoirs. However, incentives in the form of additional hydrocarbon resources (through Enhanced Oil/Gas Recovery techniques) come from using oil and gas reservoirs as storage sites which will, invariably, offset some of the cost of the sequestration process (Kalra and $\mathrm{Wu}, 2014$ ). Thus, Enhanced Gas 
Recovery (EGR) is deemed one of the potential methods for simultaneously storing anthropogenic $\mathrm{CO}_{2}$ emissions and improving additional natural gas recovery from depleted gas fields, provided that the gas miscibility in situ (mixing) can be reduced. This can be achieved by a better understanding of the mechanisms of displacement and the factors that affect them which will provide vital information for further studies aimed at a wider and robust field scale application and establish the economic viability of the process.

The adoption of EGR technique has not been generally well received. This is because of the excessive mixing of the injected carbon dioxide and in-situ natural gas during the flooding process (Al-abri et al., 2009; Honari et al., 2016, 2015, 2013; Hughes et al., 2012; Khan et al., 2013; Oldenburg and Benson, 2002; Shtepani, 2006; Sidiq et al., 2011a; Sim et al., 2008; Zhang et al., 2014). This mixing contaminates the recovered natural gas and reduces its market value by reducing its calorific value and also, incurs additional cost in the sweetening processes (Oldenburg \& Benson 2002; Sim et al. 2008; Sim et al. 2009). This necessitated an in-depth study to unearth ways to minimise this undesirable mixing phenomenon as these two gases are miscible in all ramifications. Mixing can only be minimised if the mechanics and dynamics of the process are understood. To do this, the interplay between different factors that influence the mixing of the injected $\mathrm{CO}_{2}$ and the nascent $\mathrm{CH}_{4}$ have to be investigated which will showcase the economic viability of the EGR technology. These factors stem from variations of the physical properties of the fluids, containing reservoir formations and operation conditions such as pressure, temperature, and flowrates.

Many authors (Al-abri et al., 2009; Honari et al., 2016, 2015, 2013; Hughes et al., 2012; Sidiq et al., 2011b; Sidiq and Amin, 2009) have carried out extensive researches on the sensitivity of factors, such as reservoir heterogeneity, pressure, temperature, injection rates, on the mixing between $\mathrm{CO}_{2}$ and $\mathrm{CH}_{4}$ to ascertain the influence of these investigated parameters on the gas-gas mixing during EGR. However, limited technical literatures are available on the impact of connate water saturation and salinity on $\mathrm{CO}_{2}-\mathrm{CH}_{4}$ system displacements. (Sidiq and Amin, 2009) were the only authors prior to (Honari et al., 2016) to consider connate water saturation when determining the dispersion coefficient of $\mathrm{CO}_{2}$ in $\mathrm{CH}_{4}$ in a carbon dioxide-methane systems. (Sidiq and Amin, 2009) determined the dispersion coefficient using a new model developed in their work and validated it with experimental data. However, the study was limited to the analysis of the experimental dispersion coefficients to validate the developed model and no comparative analysis was presented between saturated and dry core samples to ascertain the effects of connate water saturation on the displacement process.

A number of literatures, as reported by Honari et al. (2016), are available which considered the dispersion in a binary system comprising of different gaseous components $\left(\mathrm{N}_{2}, \mathrm{O}_{2}, \mathrm{H}_{2} \mathrm{O}\right)$ in the presence of immobile water. In this work, focus is on the experimental investigations in $\mathrm{CO}_{2}$ $\mathrm{CH}_{4}$ systems alone. Albeit not exclusively in a $\mathrm{CO}_{2}-\mathrm{CH}_{4}$ systems, Turta et al. (2007) conducted a series gas-gas displacement tests on Berea cores at a temperature of $70^{\circ} \mathrm{C}$ and a pressure of 6.2 $\mathrm{MPa}$ using Nitrogen and $\mathrm{CO}_{2}$ as injection fluids. The tests were conducted both in the presence of connate water and without connate water (dry cores) to investigate the effects of connate water on the recovery efficiency. The tests on consolidated cores showed that for pure nitrogen and pure $\mathrm{CO}_{2}$, used as the displacing fluids, the recovery was comparable. In the case where a mixture of $\mathrm{CO}_{2}$ and nitrogen were used to displace the natural gas, it was observed that there 
were delays in $\mathrm{CO}_{2}$ breakthrough, associated with a period when only a mixture of methane and nitrogen was produced. This can be attributed to the solubility of $\mathrm{CO}_{2}$ in connate water which is considerably higher than that of nitrogen. This invariably leads to a higher gas recovery due to a longer resident period, given the fact that a $20 \%$ nitrogen contamination in marketable $\mathrm{CH}_{4}$ tolerable in the produced stream, as opposed to only $2 \%$ contamination level for the case of $\mathrm{CO}_{2}$. They concluded that when using $\mathrm{CO}_{2}$ as a displacing fluid, recovery was higher in the presence of connate water saturation than in its absence invariably due to the dissolution of $\mathrm{CO}_{2}$ in the formation brine.

The first ever experimental measurement of dispersion as function of water saturation for supercritical gases in a $\mathrm{CH}_{4}-\mathrm{CO}_{2}$ system was carried out by Honari et al. (2016). They systematically measured fluid dispersion in various rock cores (sandstones and carbonates), both dry and at irreducible water saturations, at reservoir conditions. They found out that irreducible water increases dispersivity by a factor of up to 7.3. Irreducible water occupied smaller pores creating narrower pores and more tortuous flow paths giving rise to more dispersion/mixing between the injected $\mathrm{CO}_{2}$ and the in-situ $\mathrm{CH}_{4}$. Sim et al. (2009) however, inferred that the presence of irreducible water in the reservoir tends to minimise its heterogeneity and as such minimises excessive mixing as shown in their work where they used a sand pack with various degrees of permeability distributions and also a $\mathrm{N}_{2}, \mathrm{CH}_{4}, \mathrm{CO}_{2}$ binary systems.

These studies, however limited, have touched on the impact of connate water saturation on the displacement efficiency in EGR by $\mathrm{CO}_{2}$ injection. They have attributed higher dispersion coefficients to the presence of connate water. Conversely, the effect of the salinity/concentration variation of the connate water on the recovery efficiency and or dispersion coefficient was not accounted for. In this paper, the effect of connate water salinity on the dispersion coefficient was investigated experimentally in a consolidated sandstone core sample during $\mathrm{CH}_{4}$ displacement by supercritical $\mathrm{CO}_{2}$. Investigating this salinity phenomenon will help reservoir engineers better characterise gas systems for better representation in the adoption of EGR by $\mathrm{CO}_{2}$ and subsequent sequestration in natural gas reservoirs.

\subsection{Theory and concept of Enhanced gas recovery}

Dispersion is the irreversible mixing that occurs during miscible displacements (Adepoju et al., 2013), it occurs as a result of two simultaneous mechanisms; molecular diffusion and mechanical dispersion (advection) (Perkins and Johnston, 1963). When a miscible fluid displaces another miscible fluid in a porous medium, the displaced fluid tends to mix with the displacing fluid. The efficiency of local displacement in miscible flooding is grossly affected by the mixing taking place within the rock matrix. This mixing is a result of the interaction between these two fluids in contact. A transition or mixing zone develops at the displacement front where the concentration of the displacing fluid decreases from one to zero (Figure 1). It has been reported (Ekwere, 2007) that several experiments show that the mixing zone propagates longitudinally as displacement process progresses. The macroscopic mixing (dispersion) observed through porous media is used to quantify the mixing taking place (Jha et al., 2013). This can be, to an extent, analysed using empirical evaluation and laboratory experimentation as the concentration of injected $\mathrm{CO}_{2}$ relative to the in-situ $\mathrm{CH}_{4}$ in the produced effluent stream. 


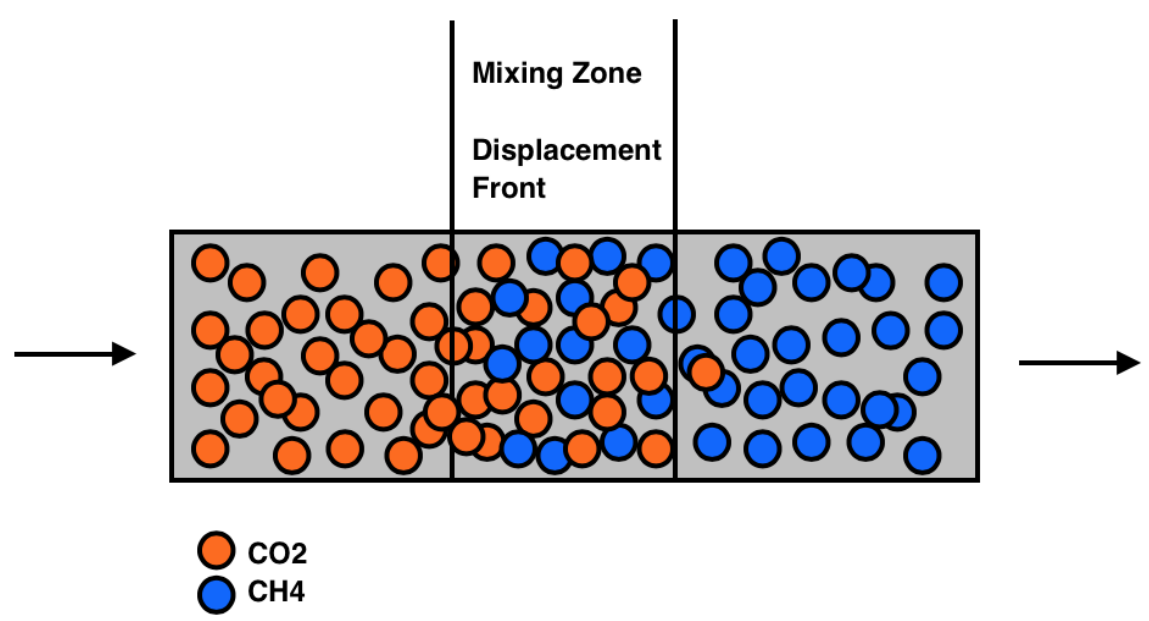

Figure 1: Schematics of displacement front during CO2 injection displacing $\mathrm{CH} 4$

In order to minimise the cost of producing the recovered natural gas and maximise potential return during enhanced gas recovery, the degree to which injected $\mathrm{CO}_{2}$ gas mixes with natural gas in situ needs to be well evaluated. Mixing has been found to be controlled by several factors including molecular diffusion/dispersion, pore geometry, turbulence, stagnant fraction of pore space, presence of an immobile fluid, viscous fingering, adsorption/desorption, and gravity segregation (Newberg and Foh, 1988). The viscosity ratio (Eq. 1) is favourable in the case of supercritical $\mathrm{CO}_{2}$ displacing $\mathrm{CH}_{4}$ because $\mathrm{CO}_{2}$ is more viscous than $\mathrm{CH}_{4}$ under reservoir conditions:

$$
\frac{\mu C H_{4}}{\mu C O_{2}}<1
$$

Gas transport in porous media occurs widely in numerous applications which include carbons sequestration, oil and gas exploitation, food processing industry etc. The importance of understanding the mechanisms of gas transport in porous media lies in allowing a number of models employed to optimise and evaluate the design and performance of the processes aforementioned.

\subsection{Application of gas transport in porous medium in EGR}

(Perkins \& Johnston, 1963) defined the mixing phenomenon occurring in porous media as a diffusion-like process due to concentration and velocity gradient. The dispersion coefficient denotes the rate of mixing when two miscible fluids come in contact at the displacement front of a flooding process. It depends on the direction of the dispersion flux with respect to the main convective flux. The smallest value of this term occurs perpendicular to the main convective path/flux often called transverse dispersion, and the largest occurs for dispersion in the main convective flux called longitudinal dispersion. Transverse dispersion coefficient, $K_{t}$, is more difficult to obtain experimentally and as result, very few data is available in literature besides those of (Perkins and Johnston, 1963).

Newberg \& Foh (1988) used a single parameter diffusion-type equation based on the AdvectionDispersion equation (Coats et al., 2009, 1964; Perkins and Johnston, 1963) which was often 
used to describe the gas transport in porous media to correlate the numerical dispersivities with experimental results and the model is as shown in (Eq. 2):

$$
K_{l} \frac{\partial^{2} C}{\partial x^{2}}-u \frac{\partial C}{\partial x}=\frac{\partial C}{\partial t}
$$

Where, $C$ is the $\mathrm{CO}_{2}$ concentration at location $x$ at time $t, K_{l}$ is the coefficient of longitudinal dispersion, and $u$ is the interstitial velocity.

This model was used to generate longitudinal dispersion coefficients and "scale of dispersion" (dispersion coefficient divided by velocity). It also describes the dispersion occurring during the displacement process in EGR.

Invariably, (Eq. 2) may be written in dimensionless form as follows (Mamora and Seo, 2002);

$$
\frac{1}{P_{e}} \frac{\partial^{2} C}{\partial x_{D}^{2}}-\frac{\partial C}{\partial x_{D}}=\frac{\partial C}{\partial t_{D}}
$$

Where;

\begin{tabular}{ccc}
\hline Parameter & Symbol & Expression \\
\hline Peclet number & $P_{e}$ & $\frac{u L}{K_{l}}$ \\
Dimensionless time & $t_{D}$ & $\frac{t u}{L}$ \\
Dimensionless distance & $x_{D}$ & $\frac{x}{L}$ \\
Interstitial velociy & $u$ & $\frac{Q}{\pi r^{2} \phi}$ \\
\hline
\end{tabular}

$L$ is length of core

$Q$ is superficial velocity,

$\phi$ is porosity

$K_{l}$ Longitudinal dispersion

Since the carbon dioxide injection inlet is at $x=0$,

then initial condition: $C=0$ at $t_{D}=0$,

boundary conditions: $C=1$ at $x_{D}=0, C \rightarrow 0$ as $x_{D} \rightarrow \infty$

The solution to (Eq. 3) maybe shown as follows:

$$
C=\frac{1}{2}\left\{\operatorname{erfc}\left(\frac{x_{D}-t_{D}}{2 \sqrt{t_{D} / P_{e}}}\right)+e^{P_{e} x_{D}} \operatorname{erfc}\left(\frac{x_{D}+t_{D}}{2 \sqrt{t_{D} / P_{e}}}\right)\right\}
$$

$\mathrm{CO}_{2}$ concentrations profiles from EGR core flooding experimentation can be compared against those based on analytic solutions of (Eq. 3) presented in (Eq. 4) for several values of Péclet number, $P_{e}$ (which is the ratio of advection to dispersion over the experimental length $L$ ) from which the corresponding dispersion coefficient can be evaluated.

(Perkins \& Johnston, 1963) presented another definition of Péclet number termed medium Péclet number denoted by, $\mathrm{P}_{\mathrm{e} m}$, which describes the dominant displacement regime during a dispersion process and expressed in (Eq. 5) as:

$$
P_{e m}=\frac{u_{m} d}{D}
$$


Where $P_{e m}$ is medium Péclet number, $u_{m}$ is the mean interstitial velocity $(\mathrm{m} / \mathrm{s}), D$ is the diffusion coefficient $\left(\mathrm{m}^{2} / \mathrm{s}\right)$, and $d$ is the characteristic length scale of the porous medium. Generally, at $\mathrm{P}_{\mathrm{e} m}$ $<0.1$, diffusion dominates the dispersion process and the ratio, is constant and equates to and conversely, at $\mathrm{P}_{\mathrm{e} m}>10$ advective mixing dominates the dispersion process and the ratio linearly proportional to $\mathrm{P}_{\mathrm{e} m}$. And in this range of $\mathrm{P}_{\mathrm{e} m}$, (Coats et al., 2009) correlated dispersion coefficient with diffusion shown in (Eq. 6):

$$
\frac{K_{l}}{D}=\frac{1}{\tau}+\alpha \frac{u_{m}^{n}}{D}
$$

Where $\alpha$ is in $m$ and is the dispersivity of the porous medium, $n$ is an exponent. Which is and $\tau$ can range from for packed beds and can be as large as 13 for consolidated media as reported by (Honari et al., 2013) and literatures therein. The parameter $\tau$ can be obtained empirically through several methods, but $\alpha$ and $n$ can only be determined experimentally through core flooding (Hughes et al., 2012).

Furthermore, Takahashi and Iwasaki 1970, as reported by (Hughes et al., 2012) and (Liu et al., 2015), established a correlation between the molecular diffusion coefficient, temperature and pressure. This correlation was used by the authors to obtain accurate diffusivity using (Eq. 7) at conditions relevant to enhanced gas recovery by $\mathrm{CO}_{2}$ injection. The correlation is as follows:

$$
D=\frac{\left(-4.3844 \times 10^{-13} p+8.55440 \times 10^{-11}\right) T^{1.75}}{p}
$$

where $D\left(\mathrm{~m}^{2} / \mathrm{s}\right)$ is the molecular diffusion coefficient of $\mathrm{CO}_{2}$ in $\mathrm{CH}_{4}$ at temperature $\mathrm{T}(\mathrm{K})$ and pressure $p(\mathrm{MPa})$. In the works of Takahashi and Iwasaki, the diffusion coefficients of $\mathrm{CO}_{2}$ in $\mathrm{CH}_{4}$ were measured at 298 to $348 \mathrm{~K}$ and pressures of 5-15 $\mathrm{MPa}$ in a porous bronze plug which are well within the range of conditions applicable to EGR.

\section{Experimental methodology}

\subsection{Materials used}

Pure methane with a purity of $99.995 \%$ and research grade carbon dioxide with a purity of 99.999\% were supplied by BOC UK a member of the Linde Group. The core sample was obtained from Kocurek Industries USA. The salts employed in this research were supplied by Fisher Scientific UK. The petrophysical properties of the core sample used are shown in Table 1. The porosity was evaluated experimentally using Helium Porosimetry technique.

\subsection{Apparatus and procedure}

\subsubsection{Helium Porosimetry}

This method facilitates the determination of the grain volume of a core sample which is volume of the rock grains or solids alone. The pore volume was then determined from the difference between the grain volume obtained by this method and the bulk volume which is defined as the volume the sample occupies. The Bulk Volume is determined empirically and analytically by measuring the dimension of the core samples using a high accuracy Vernier caliper and using the cylinder volume determination formulation.

$$
\text { Pore Volume }=\text { Bulk Volume - Grain Volume }
$$


Where, Bulk Volume $=\frac{\pi d^{2}}{4} \times L$, and $\mathrm{d}$ is the core diameter, and $\mathrm{L}$ is core length.

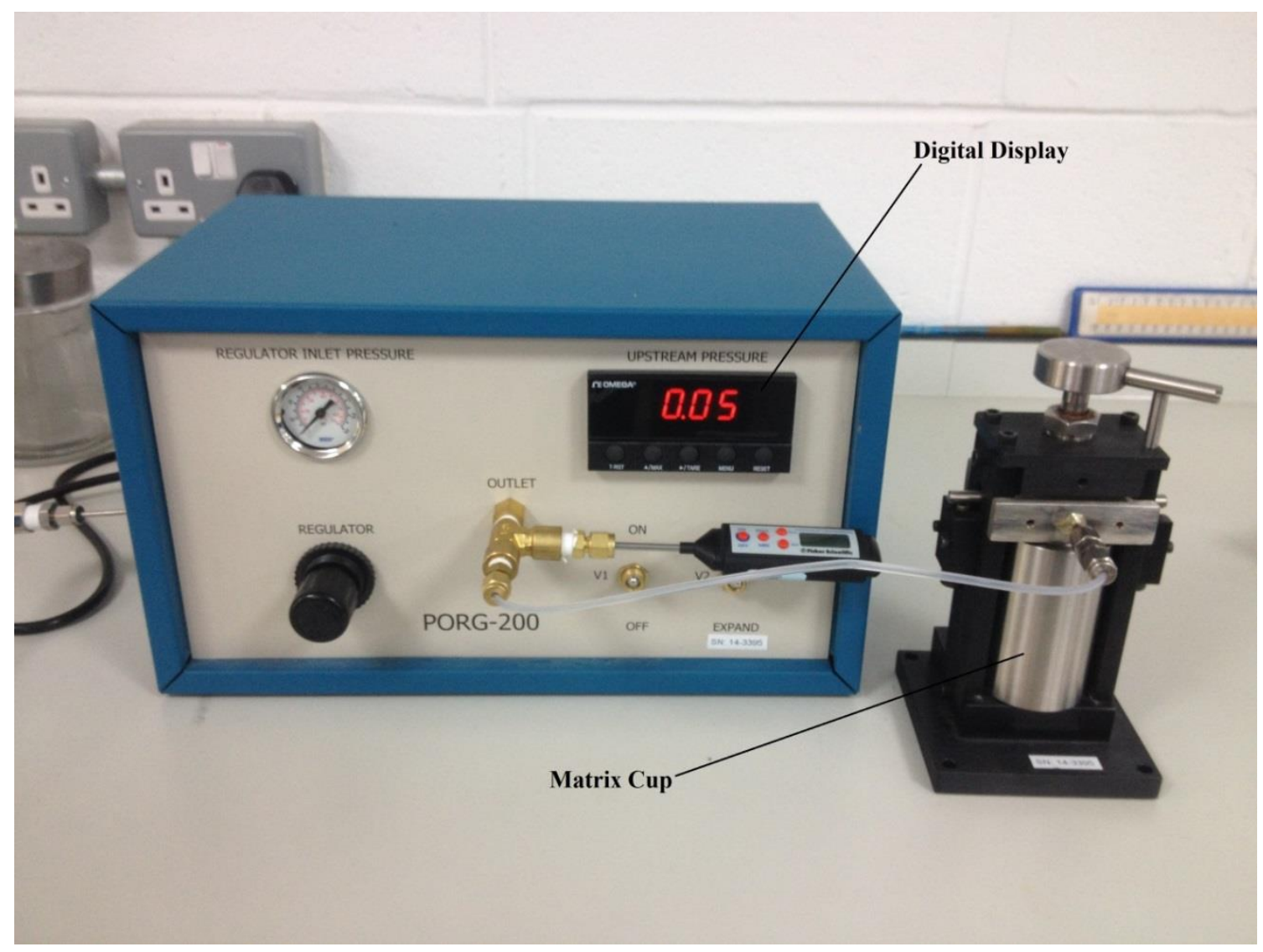

Figure 2: PORG-200 porosimeter

\subsubsection{Procedure}

The Helium gas supply was connected to the gas inlet port of the instrument and was set to 120 psig on the Helium gas bottle regulator. After a leak test, the system grain volume calibration was performed on the Porosimeter. The matrix cup with reference discs was then connected to the instrument. The sample grain volume measurement was performed, and the obtained results were recorded in the provided application written in excel spreadsheet which evaluated the grain volume of each sample. 
Table 1: Petrophysical properties of the core sample

\begin{tabular}{ccccc}
\hline $\begin{array}{c}\text { Core } \\
\text { sample }\end{array}$ & $\begin{array}{c}\text { Length } \\
(\mathbf{m m})\end{array}$ & $\begin{array}{c}\text { Diameter } \\
(\mathbf{m m})\end{array}$ & $\begin{array}{c}\text { Porosimetry } \\
\text { Porosity } \\
(\boldsymbol{\%})\end{array}$ & $\begin{array}{c}\text { Permeability } \\
(\mathbf{m D})\end{array}$ \\
\hline Grey Berea & 76.27 & 25.22 & 20 & 217 \\
\hline
\end{tabular}

\subsubsection{Core flooding equipment description}

The core flooding equipment used is a branded system by CoreLab Oklahoma, USA. The equipment was modified to carryout gas-gas displacement processes by integrating an Agilent Gas Chromatograph 7890A for effluent analysis depicted in Figure 3. The core flooding system was rated to 5,000 psig confining pressure, 3,500 psig pore pressure at room temperature. The inlet pressure into the core sample and outlet pressures on the other side of each core are measured with gauge pressure transducers. An integral part of the system is the SmartFlood software and computer data-acquisition-and-control system hardware which provides on-screen display of all measured values (pressures, temperatures, volumes etc.), automatic logging of test data to a computer data file. The core sample is held within a rubber sleeve inside a Hassler-type core holder by radial confining pressure, which simulates reservoir overburden pressures. The simulated pore pressure was applied through a ISCO model 500D, two-barrel metering pump system with a flow rate range adjustable from 0 to $200 \mathrm{ml} / \mathrm{min}$ and a maximum pressure rating of $4,000 \mathrm{psig}$. The overburden (confining) pressure pump is a hydraulic pump Model S-216-JN-150 pump, with pressure output of up to 10,000 psig and will provide the desired overburden in the system. The back pressure is regulated with CoreLab dome-loaded type back-pressure regulator which controls the back pressure to a reference pressure supplied to its dome. It is rated for a maximum working pressure of 5,000 psig. Floating-piston accumulators are provided as part of the system and are rated for $5,000 \mathrm{psig}$ pressure and $350^{\circ} \mathrm{F}\left(177^{\circ} \mathrm{C}\right)$ temperature. The accumulators provide for injecting fluids without allowing the fluid to come in contact with the metering pump. 2.5-inch-dial pressure gauges are used to monitor the Overburden Pressure and the BPR Dome Pressure. The pressure range on these gauges is 15,000-psig full scale. Rosemount transducer provided with the system measure differential pressure across the core holder. The effluent flowrate and produced volume was measured by Bronkhorst mass flow controllers/meters and records the effluent rates on the logging worksheet of the SmartFlood software. The picture and schematic of the equipment are presented in Figure 3 and Figure 4. 


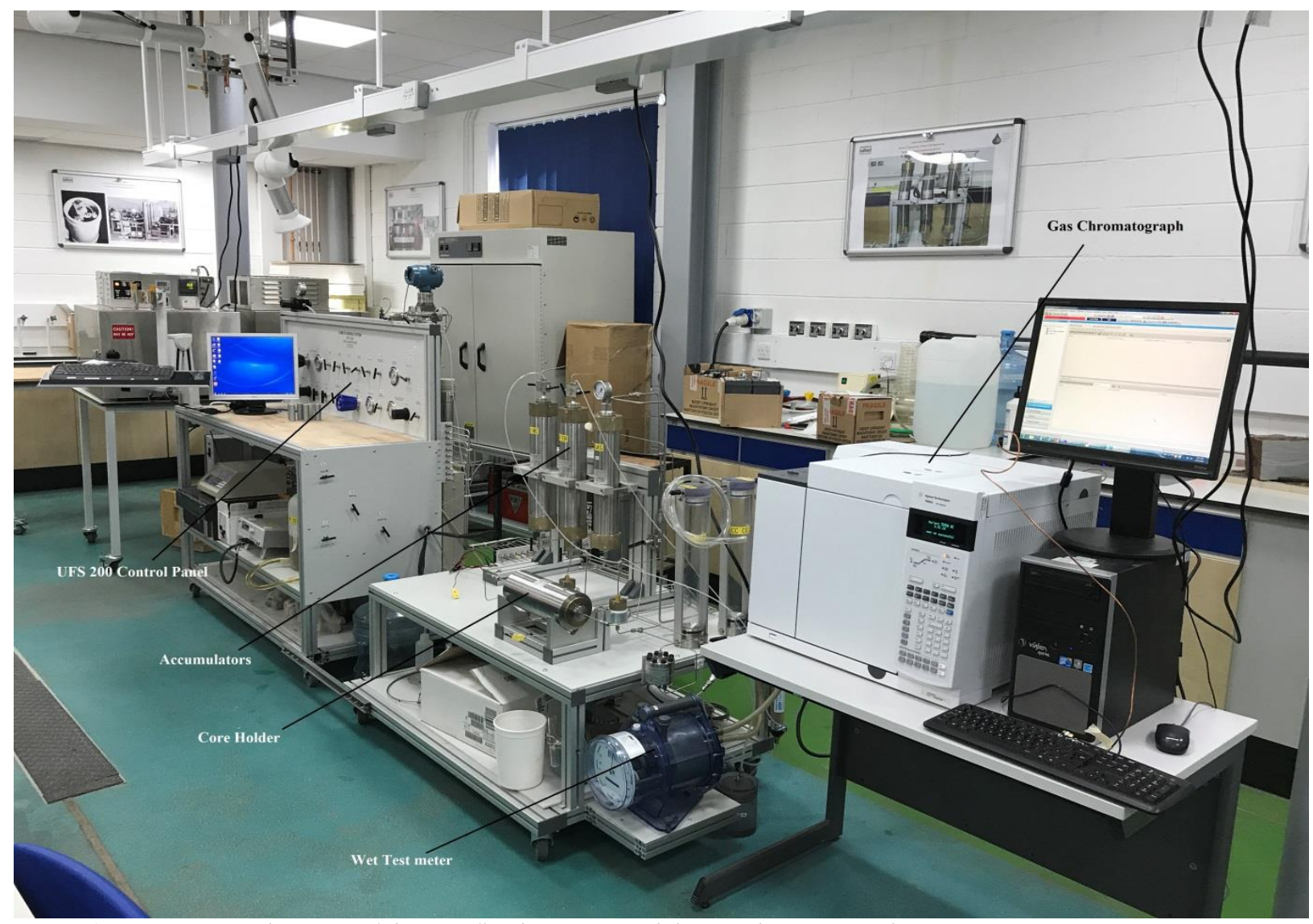

Figure 3: A perspective depiction of the core flooding setup and the gas chromatogragh 


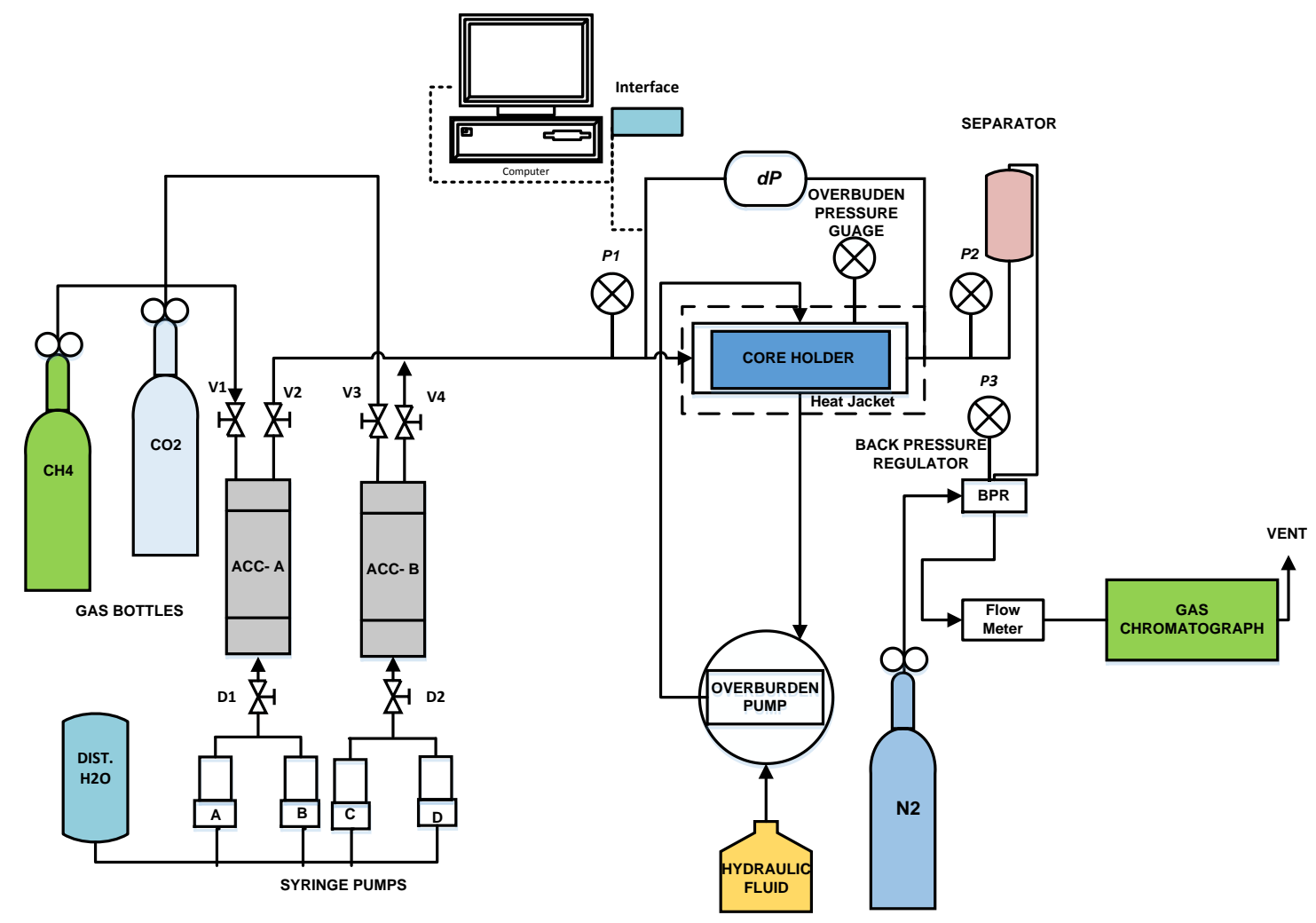

Figure 4: Schematics of core flooding set up

\subsubsection{Procedure}

\subsection{Salt preparation and core sample saturation}

The $\mathrm{NaCl}$ salt was measured and dissolved in distilled water contained in a round bottom flask with a magnetic stirrer to prepare the desired brine concentrations of 5 and $10 \mathrm{wt} \%$. The core sample was saturated with $10 \%$ of its pore volume (determined from Helium porosimetry) with the brine of the concentrations as aforementioned using a vacuum technique. Initial dry runs were first carried out to ascertain repeatability of the set up and method. Then wet runs were performed subsequently, first using distilled water to establish a datum for the salinity variation experiments and then using the prepared concentrations of brine.

\subsection{Core flooding}

The core sample was wrapped in foil paper to avoid the permeation of the supercritical gases through the sleeve and into the annulus of the core holder. A layer of cling film was first placed between the core sample and the foil paper to prevent the foil paper from sticking to the core sample when subjected to high temperatures and pressures. The core sample was then placed inside the Viton sleeve and installed on the distribution plugs of the core holder and secured with clamps on both sides and inserted into the core holder. A heat jacket was placed around the core holder and the temperature ramping was set and the hydraulic pump was initiated to pump the hydraulic oil into the annulus of the core holder to provide the overburden pressure necessary for the experiment in lieu of the simulated reservoir depth pressure. A pressure of 2200 psig was set 
as the overburden pressure. The simultaneous hydraulic oil pumping and heating was done to avoid high temperature ramping with uncontrolled pressure rise. When the core holder temperature reached $50^{\circ} \mathrm{C}$, the temperature ramping was stopped, and the temperature was kept constant. Hydraulic oil leaks were checked for on both sides of the core holder to ensure that the clamping of the core sample and set up integrities were not compromised.

As a safety precaution, all the valves were shut off. V1 was opened to provide access to the accumulator. D1, depicted in the schematics in Figure 4, was opened to purge pumps A and B to give room for filling the accumulator A, ACC-A, with the $\mathrm{CH}_{4}$ gas from the bottle. When there was no increment in the level of the distilled water in the reservoir, D1 was shut off and then V1 was shut off too. The back pressure reference pressure was set to 1300 psig using the $\mathrm{N}_{2}$ gas bottle. The $\mathrm{N}_{2}$ gas was used to set the dome pressure of the back pressure regulator as opposed to the hydraulic oil because of the compressibility of the gas which provided a smoother flow of the gas and avoided pressure build-up within the core flooding and the reference pressure was kept constant. V2 was then opened to saturate the system with $\mathrm{CH}_{4}$. Pumps A\&B were engaged to compress the gas in the system to provide the desired system pressure. V2 was then shut off.

The same filling procedure was carried out with accumulator B, ACC-B. V4 was then opened and then the logging commenced and also the GC sequence as run. The items logged were differential pressure, $\mathrm{dP}$, production rate, each time stamp was recorded which corresponded with the injection times of the GC, whose method sequentially runs for five (5) minutes to sample the effluent every five minutes. The flowrate was measured with the flow meters. The overburden pressure was carefully monitored and was kept more than 500 psig above the pore pressures to avoid the rupturing of the vitton sleeve, given that the pumps deliver a constant flowrate and the pressures rapidly build to maintain the desired flowrate.

Each experimental run came to an end when there were insignificant volumes of $\mathrm{CH}_{4}$ in the effluent analysis from the GC.

\section{Results and discussion}

\subsection{Optimum injection rate determination}

In order to carry out the investigation on the salinity effects on dispersion coefficient, an optimum injection rate for this case was determined. Here, an experimental screening was carried out based on the dispersion coefficient and the $\mathrm{CH}_{4}$ recovery efficiency to evaluate the optimum injection rate from a range of experimental values $-0.2-0.5 \mathrm{ml} / \mathrm{min}$ adopted from literature (Liu et al., 2015). This is to ascertain the best case to evaluate the effects of the connate water salinity during flooding and to minimise other systematic effects emanating from the variation in interstitial velocity within the pore matrix. This systematics, as shown in literatures cited within this work, have their various effects on dispersion coefficient and the rock dispersivity.

\subsubsection{Dispersion coefficients and dispersivity}

A number of displacement runs were carried out to check for consistency and repeatability of the experimental set-up using the same core sample under dry conditions. The evaluated $K_{L}$ for the test runs are shown in Table 2 . When the results became consistent by adjusting the experimental methodology and set-up, the different flowrates were then employed to determine the optimum injection rate from the range of interest. The concentration profiles were used to evaluate the rate 
of mixing of the injected $\mathrm{CO}_{2}$ and the nascent $\mathrm{CH}_{4}$ using (Eq. 3) as aforementioned and adopting the longitudinal dispersion coefficient $K_{L}$ as the fitting parameter. The values of the dispersion coefficients for different injection rates are shown in Table 3. The fitted graph of the different injection rates is shown in Figure 7 . The $L$ was adjusted in the regression to provide a better fit as advised by (Hughes et al., 2012) and (Liu et al., 2015) given that the interstitial velocity was held constant as assumed in the 1D advection dispersion equation (Eq. 2). Least square regression method was employed in the curve fitting technique. As expected, the higher injection rates showed early breakthrough of the $\mathrm{CO}_{2}$ which is in agreement with the works of (Liu et al., 2015). This also shows that the higher the injection rate the higher the dispersion coefficient as seen in Figure 5 which showcases the relationship between the two parameters.

Table 2: Dispersion coefficient determination for test runs

\begin{tabular}{cccccc}
\hline Runs & $\begin{array}{c}\mathbf{Q} \\
(\mathbf{m l} / \mathbf{m i n})\end{array}$ & $\left.\begin{array}{c}\mathbf{u} \\
(\mathbf{1 0}\end{array} \mathbf{~ m} / \mathbf{s}\right)$ & $\begin{array}{c}\text { Pressure } \\
(\mathbf{p s i g})\end{array}$ & $\begin{array}{c}\text { Temperature } \\
(\mathbf{0} \mathbf{C})\end{array}$ & $\begin{array}{c}\mathbf{K}_{\mathbf{L}} \\
\left(\mathbf{1 0}^{-\mathbf{8}} \mathbf{~ m}^{\mathbf{2}} / \mathbf{s}\right)\end{array}$ \\
\hline Run 1 & 0.25 & 3.45 & 1300 & 50 & 1.989 \\
Run 2 & 0.25 & 3.45 & 1300 & 50 & 4.125 \\
Run 3 & 0.25 & 3.45 & 1300 & 50 & 8.732 \\
Run 4 & 0.25 & 3.45 & 1300 & 50 & 2.681 \\
Run 5 & 0.25 & 3.45 & 1300 & 50 & 2.849 \\
Run 6 & 0.25 & 3.45 & 1300 & 50 & 2.206 \\
Run 7 & 0.25 & 3.45 & 1300 & 50 & 2.848 \\
Run 8 & 0.25 & 3.45 & 1300 & 50 & 2.452 \\
\hline
\end{tabular}

Furthermore, the $P_{e m}$ was evaluated using (Eq. 5), in that the characteristic length scale of mixing, $d$, was evaluated by measuring the mean grain diameter of the core sample using a novel experimental method which will be presented in a subsequent paper. This value was found to be $94 \mu \mathrm{m}$. This was then used to determine the dominant mechanism of displacement i.e. the value of the Peclet number which was 0.018 meaning that diffusion is the dominant displacement mechanism in the experimental run. 


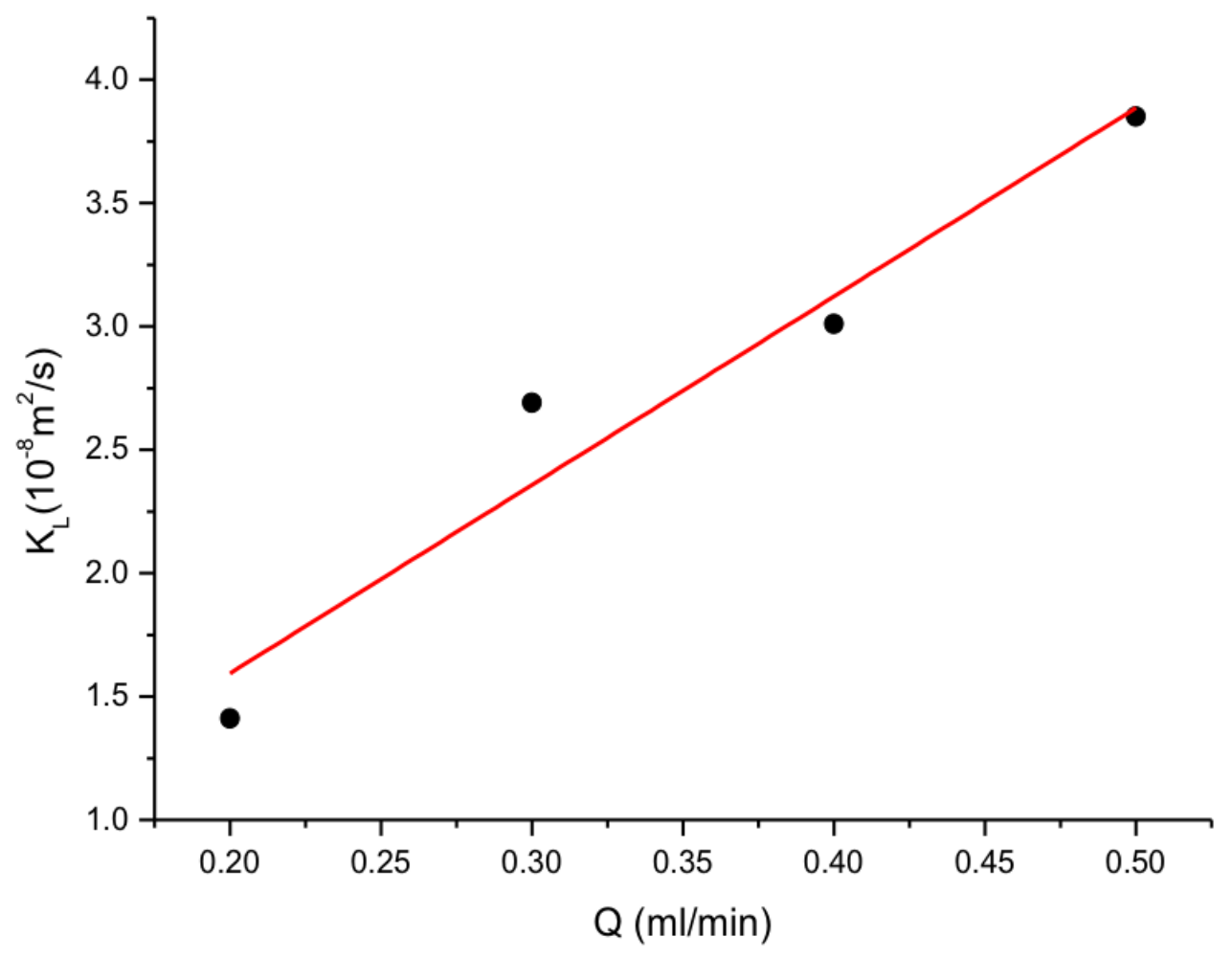

Figure 5: Variation of Dispersion coefficient with injection rate

The dispersivity was also evaluated using the relation in (Eq. 6) where the ratio k/D was plotted against the ratio u/D which is a straight, shown in Figure 6, and the gradient/slope represented the parameter. The value of the dispersivity was $0.0006 \mathrm{~m}$ which is well within the range obtained by (Hughes et al., 2012) for consolidated porous media. 


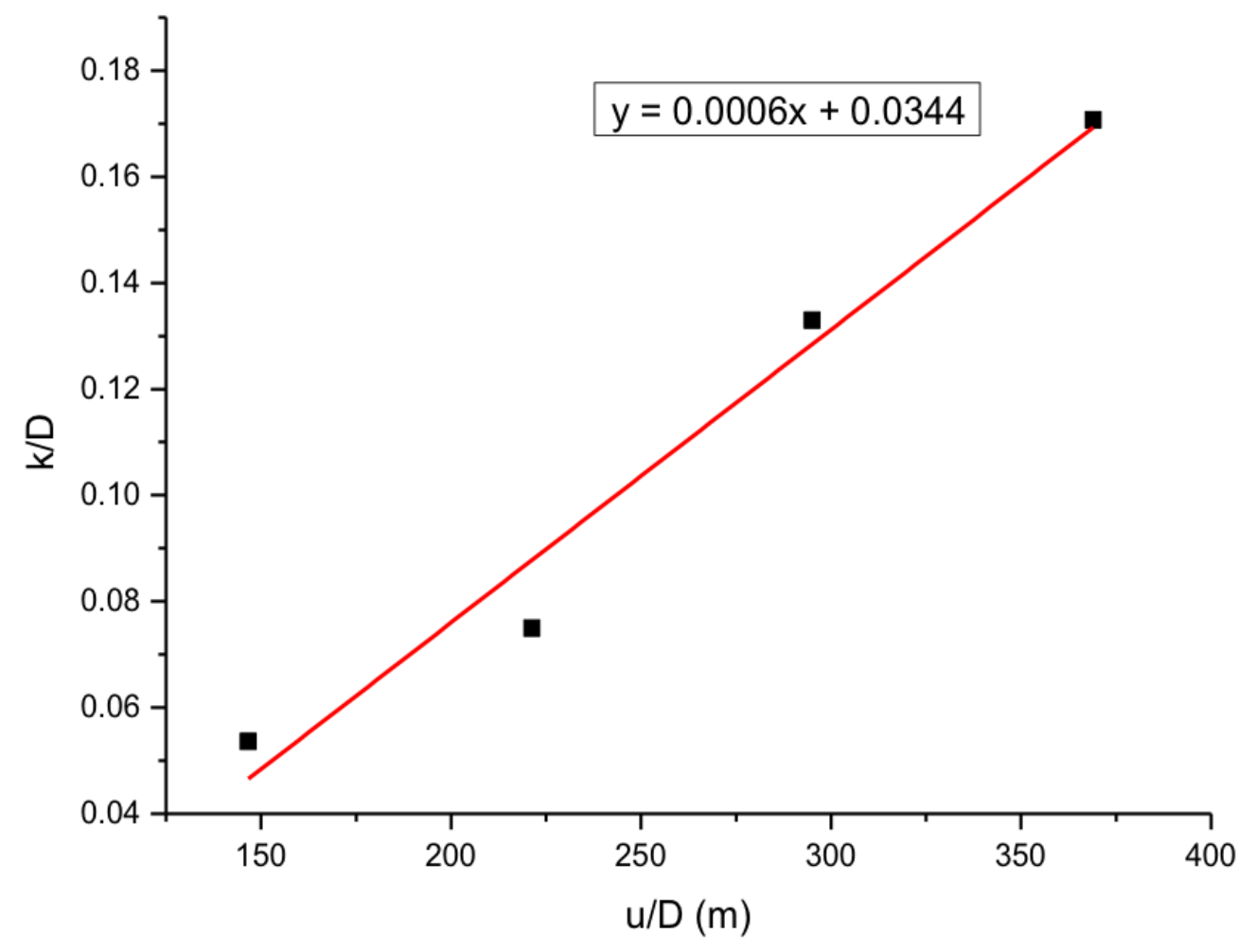

Figure 6: Dispersity of the core sample at test conditions 


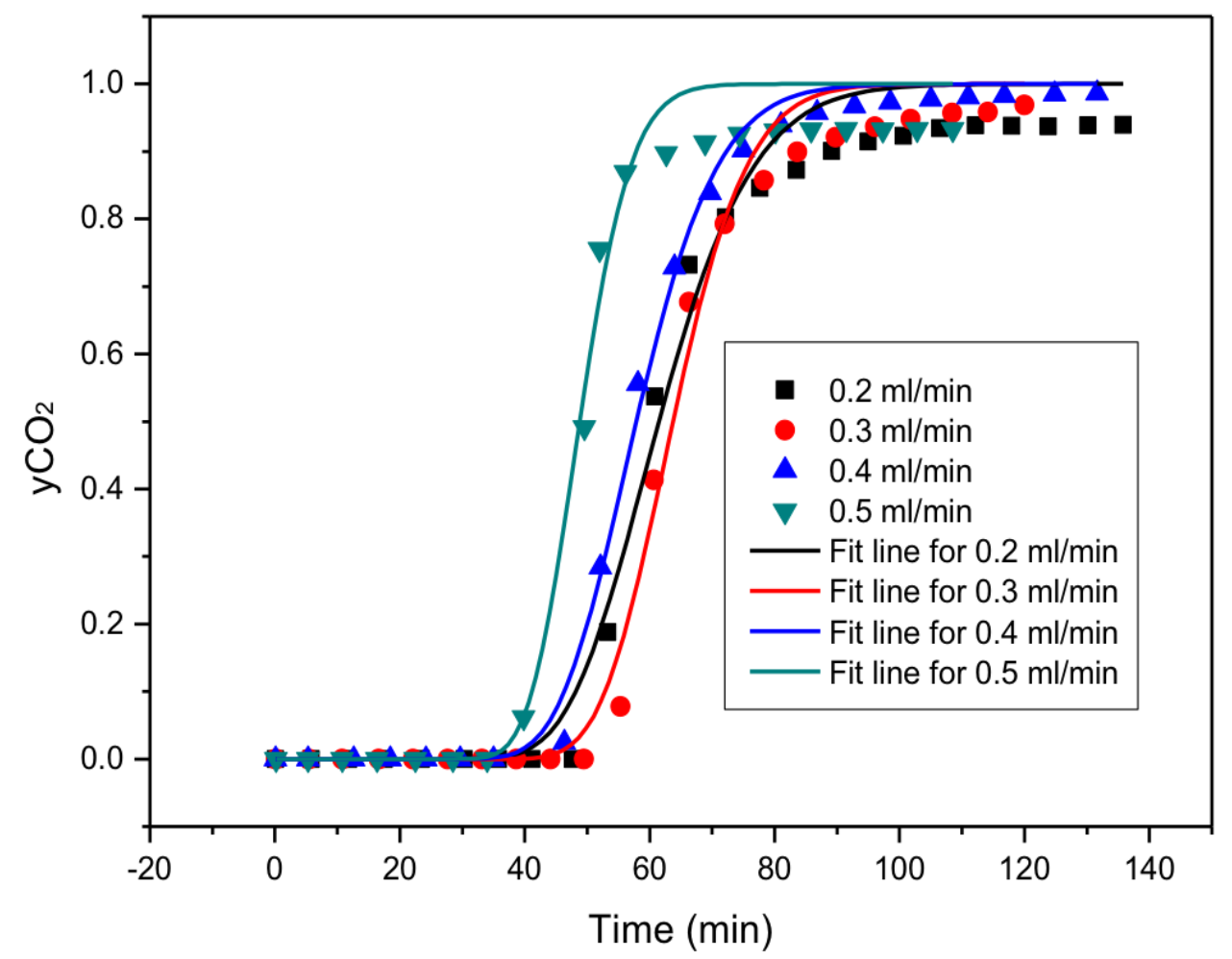

Figure 7: Fitted concentration profiles of different experimental runs

Table 3: Dispersion coefficient determination for different injection rates

\begin{tabular}{cccccc}
\hline $\begin{array}{c}\mathbf{Q} \\
(\mathbf{m l} / \mathbf{m i n})\end{array}$ & $\begin{array}{c}\text { Pressure } \\
(\mathbf{p s i g})\end{array}$ & $\begin{array}{c}\text { Temperature } \\
\left({ }^{\mathbf{0}} \mathbf{C}\right)\end{array}$ & $\begin{array}{c}\mathbf{u} \\
\left(\mathbf{1 0}^{-5} \mathbf{m} / \mathbf{s}\right)\end{array}$ & $\begin{array}{c}\mathbf{K}_{\mathbf{L}} \\
\left(\mathbf{1 0}^{\mathbf{8}} \mathbf{m}^{\mathbf{2}} / \mathbf{s}\right)\end{array}$ & $\begin{array}{c}\mathbf{D} \\
\left(\mathbf{1 0}^{-\mathbf{8}} \mathbf{m}^{\mathbf{2}} / \mathbf{s}\right)\end{array}$ \\
\hline 0.2 & 1300 & 50 & 3.31 & 1.41 & 22.56 \\
0.3 & 1300 & 50 & 5.01 & 2.69 & 22.56 \\
0.4 & 1300 & 50 & 6.66 & 3.01 & 22.56 \\
0.5 & 1300 & 50 & 8.33 & 3.85 & 22.56 \\
\hline
\end{tabular}




\subsubsection{Optimum $\mathrm{CH}_{4}$ recovery efficiency}

To determine the percentage recovery of the $\mathrm{CH}_{4}$, the original gas in place OGIP was determined using (Eq. 9). The porosity obtained from the He porosimetry, the Gas formation volume factor was calculated at the experimental conditions with the compressibility factor, $Z$, obtained numerically from the models in works of (Shabani and Vilcáez, 2017; Ziabakhsh-Ganji and Kooi, 2012) which provided a better presentation of the parameter.

$$
G=\frac{v_{b} \phi\left(1-S_{w}\right)}{B_{g}}
$$

The value of the OGIP was then used to calculate the $\mathrm{CH}_{4}$ percentage recovery using the production rates obtained from the mass flow meters of the core flooding set-up. $\mathrm{CH}_{4}$ production recovery, expressed as pore volumes produced, was evaluated and plotted as a function of time which as shown in Figure 8.

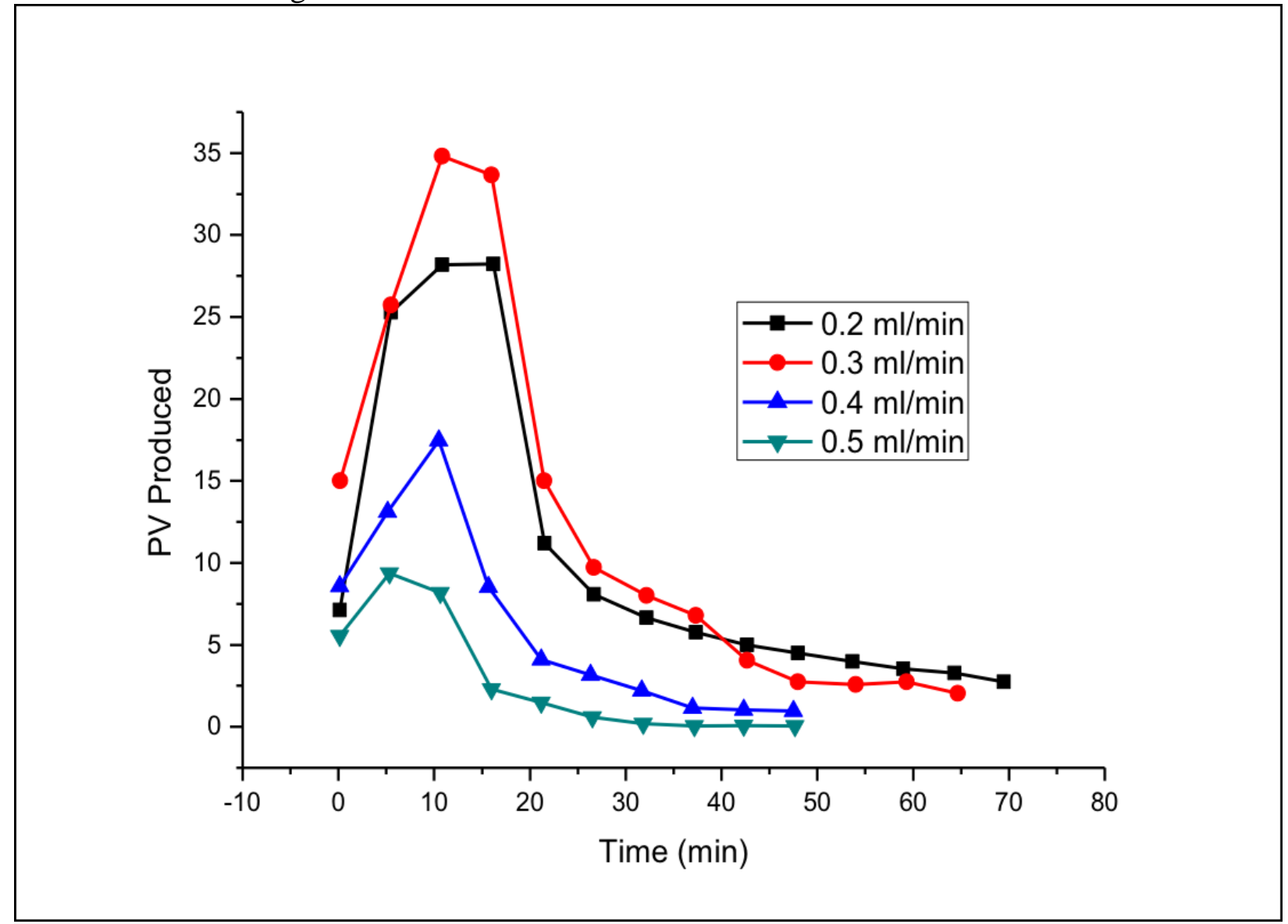

Figure 8: $\mathrm{CH}_{4}$ recovery as a function of time

Figure 8 is a representation of the results of the $\mathrm{CH}_{4}$ production recovery efficiency obtained from the core flooding experiments using different injection rates at the same reservoir conditions. Each run has a characteristic peculiarity and trend. For the experimental run at 0.2 $\mathrm{ml} / \mathrm{min}$, the recovery was substantial but the resident time for the displacement was longer and hence a stream of $\mathrm{CH}_{4}$ laced or contaminated by the $\mathrm{CO}_{2}$ was recovered. In that, there was 
substantial mixing between the displaced and displacing gases given the nature of the miscibility between them albeit having lower dispersion coefficient. This is not conceivably an economic derivative as more $\mathrm{CH}_{4}$ will be produced which will be grossly contaminated by the injected $\mathrm{CO}_{2}$ thereby undermining the sequestration idea.

Consequently, the experimental run at $0.3 \mathrm{ml} / \mathrm{min}$ showcases a different scenario with the highest recovery trend in all the experimental runs. There was a substantial $\mathrm{CH}_{4}$ recovery and good sweep efficiency compared to the runs of $0.4 \mathrm{ml} / \mathrm{min}$ and $0.5 \mathrm{ml} / \mathrm{min}$ which show a very poor trend in terms of $\mathrm{CH}_{4}$ recovery and sweep efficiency as a result of higher interstitial velocity. High interstitial velocities tend to increase the turbulence of the flow profile and agitate the molecules of the gas species which in turn facilitates the interaction between the displacing and displaced fluids.

With the results from the dispersion coefficient determination and $\mathrm{CH}_{4}$ recovery efficiency, it is apparent that the best and optimum injection rate for $\mathrm{CO}_{2}$ for this experiment is the $0.3 \mathrm{ml} / \mathrm{min}$. Thus, this flow rate will be adopted in the main experiment to investigate the effect of connate water salinity on dispersion.

\subsection{Connate water salinity investigation}

Having determined the optimum $\mathrm{CO}_{2}$ injection rate, the next step was to investigate the effect of connate water salinity on dispersion coefficient using the obtained injection rate. The connate water saturation was set to $10 \%$ to establish an immobile phase at the operating conditions based on the size and pore geometry of the core sample. This was done by saturating the core sample with $10 \%$ of its pore volume with distilled water, brine (5 wt $\%)$, and brine (10 wt $\%$ ) under vacuum for effective distribution throughout the pore matrix of the core sample.

The dispersion coefficient of each saturation and salinity will be highlighted to evaluate the effect of the both parameters on the mixing during EGR.

\subsubsection{Dispersion coefficient measurement}

Table 4 shows the results obtained from curve fitting the experimental data obtained from core flooding at different salinities but at the same operation conditions.

Table 4: Dispersion coefficient as a function of salinity

\begin{tabular}{cccccc}
\hline Run & $\mathbf{S}_{\text {wi }}(\boldsymbol{\%})$ & $\begin{array}{c}\text { Salinity } \\
(\mathbf{w t} \%)\end{array}$ & $\begin{array}{c}\text { Pressure } \\
(\mathbf{p s i g})\end{array}$ & $\begin{array}{c}\text { Temperature } \\
\left({ }^{\mathbf{0}} \mathbf{C}\right)\end{array}$ & $\mathbf{K}_{\mathbf{L}}\left(\mathbf{1 0}^{-\mathbf{8}} \mathbf{m}^{\mathbf{2}} / \mathbf{s}\right)$ \\
\hline 1 & 10 & 10 & 1300 & 50 & 0.44 \\
2 & 10 & 5 & 1300 & 50 & 0.59 \\
3 & 10 & 0 & 1300 & 50 & 3.61 \\
4 & 0 & 0 & 1300 & 50 & 2.82 \\
\hline
\end{tabular}

The results are consistent with the finding of (Abba et al., 2017) who carried out the investigation at a temperature of $40^{\circ} \mathrm{C}$ and a pressure of $1300 \mathrm{psig}$. They explained the trend observed was as a result of reduction in the tortuous flow paths of the porous medium with connate water inclusion in the experimental run. However, when distilled water was used, the distribution of the water in the pore matrix did not completely seal off smaller pores but instead 
reduced the pore throats resulting in narrower flow paths and hence higher interstitial velocities. Here, the dispersion coefficient was highest, which was attributed that the low density of the connate water, compared to the brines, was responsible for higher dispersion coefficient observed.

The fitted curves of the concentration profiles are shown in Figure 9. Early breakthrough of $\mathrm{CO}_{2}$ was apparent in the runs with saturations of $10 \%$ by volumes, given that the pore volume of the core sample was reduced by $10 \%$ due to the inclusion of connate water.

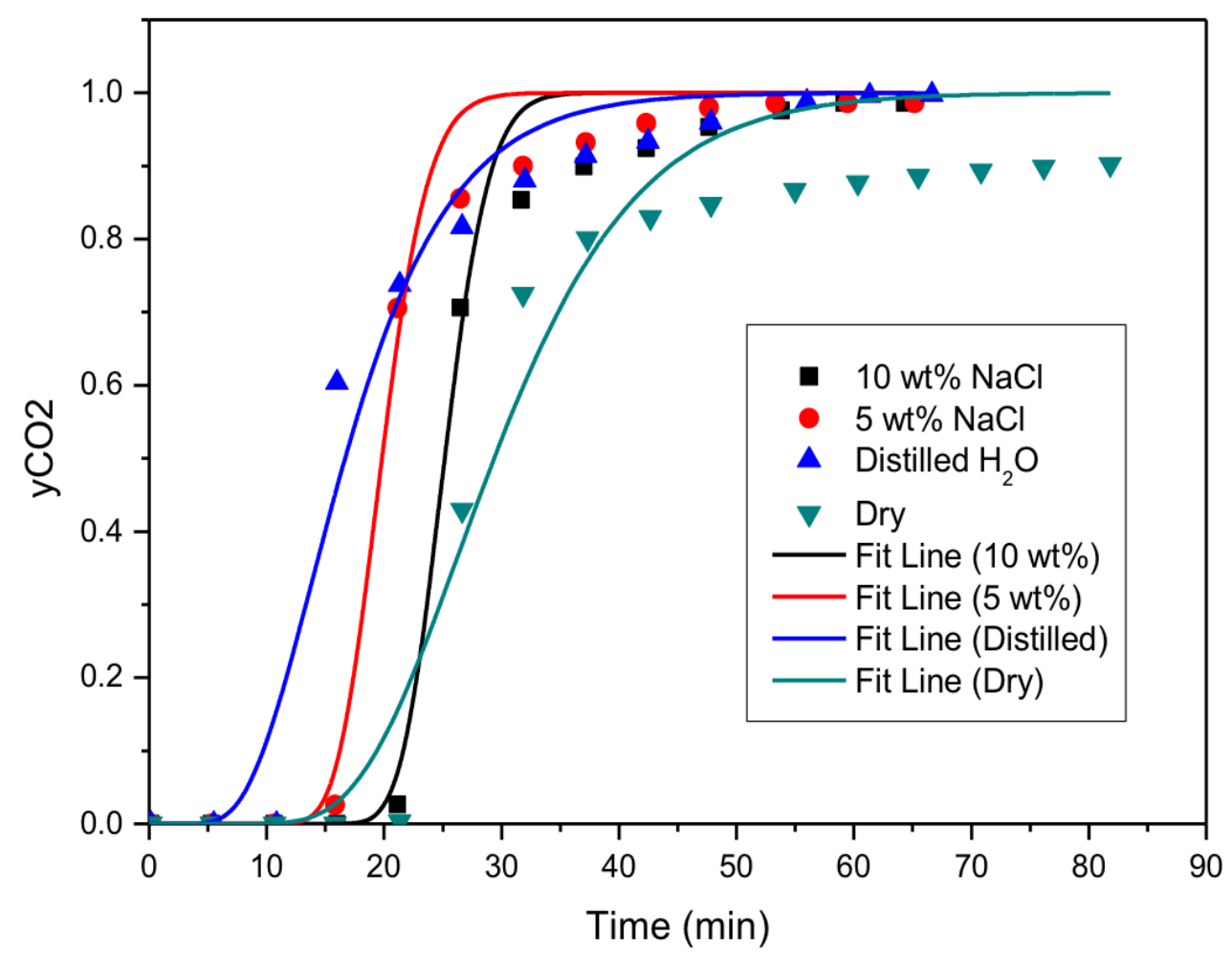

Figure 9: Concentration profiles of different variants of salinities and air at test conditions

There was meagre fitting of the analytical solution to the experimental data at the tail end of the concentration profiles and this was as a result of the entry and exit effects of the displacing supercritical $\mathrm{CO}_{2}$ which was pointed out by (Honari et al., 2013; Hughes et al., 2012) and reiterated by (Liu et al., 2015). Details of this tailing effect are presented in the (Liu et al., 2015).

The densities of the different connate water salinities were simulated and shown in Figure 10 using PVTsim 20. This was carried out to highlight the interplay between the formation water salinities and the dispersion coefficient. This relationship between the connate water salinity and the dispersion coefficient is first shown in this body of work to the knowledge of this research. 


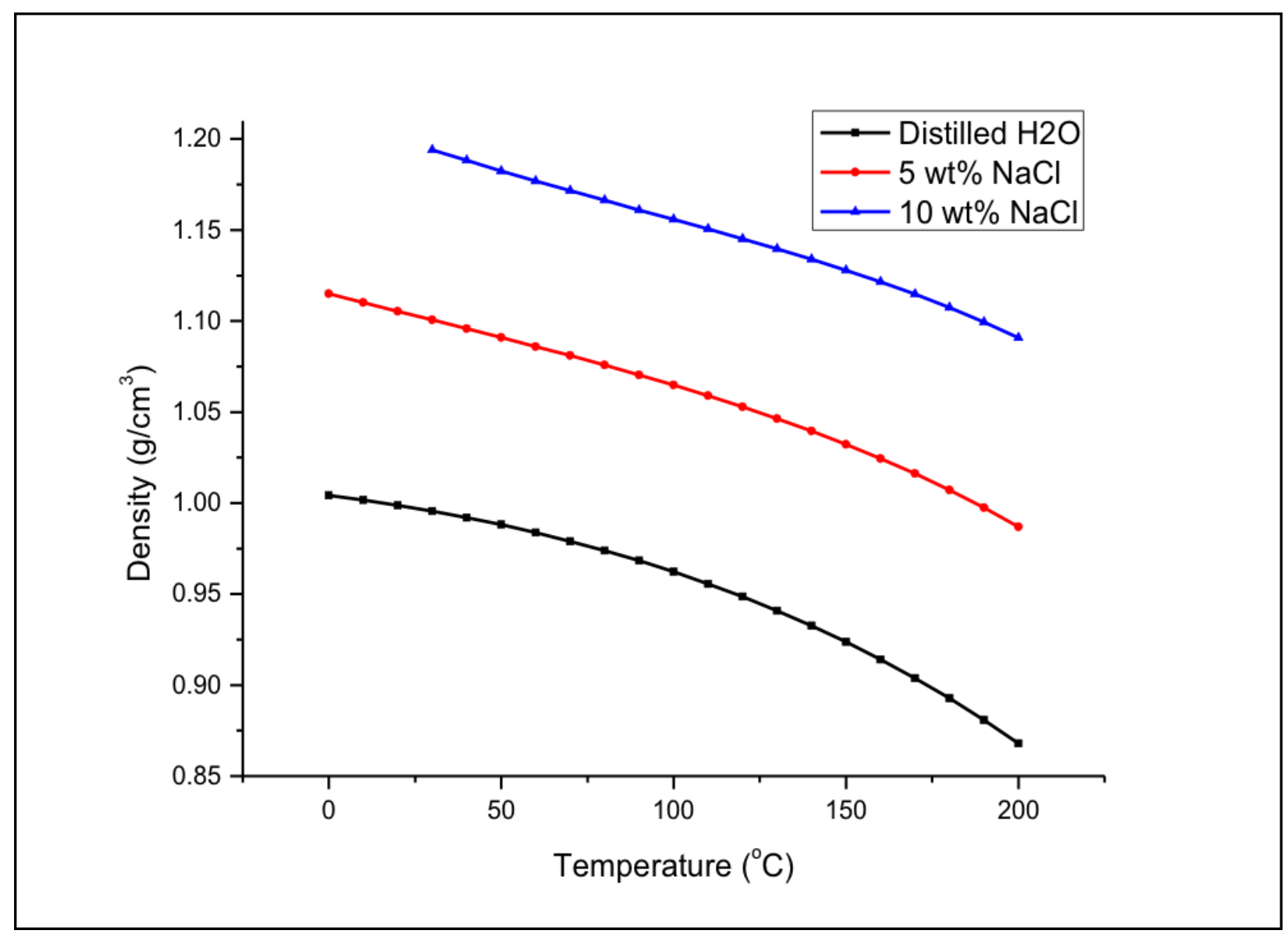

Figure 10: Connate water densities as functions of temperature at 1400 psig (Generated from PVTsim 20)

From the simulation results, the densities were extracted at the desired conditions and tabulated below in Table 5 .

Table 5: Brine concentrations with corresponding densities

\begin{tabular}{|c|c|c|c|}
\hline Salinity (wt\%) & Temperature $\left({ }^{\circ} \mathrm{C}\right)$ & Pressure (psig) & Density $\left(\mathrm{g} / \mathrm{cm}^{3}\right)$ \\
\hline 10 & 50 & 1300 & 1.18245 \\
\hline 5 & 50 & 1300 & 1.09095 \\
\hline 0 & 50 & 1300 & 0.98796 \\
\hline
\end{tabular}

Table 6: Fluid densities with corresponding dispersion coefficients

\begin{tabular}{cccc}
\hline Run & Salinity $(\mathbf{w t} \%)$ & Density $\left(\mathbf{g} / \mathbf{c m}^{\mathbf{3}}\right)$ & $\mathbf{K}_{\mathbf{L}}\left(\mathbf{1 0}^{\mathbf{- 8}} \mathbf{~ m}^{\mathbf{2}} / \mathbf{s}\right)$ \\
\hline 1 & 10 & 1.18245 & 0.44 \\
2 & 5 & 1.09095 & 0.59 \\
3 & 0 & 0.98796 & 3.61 \\
\hline
\end{tabular}


Properties tabulated in Table 6 clearly show the observed relationship between connate water densities and the longitudinal dispersion coefficients. The postulate that as the density of the connate water in the pore spaces of the core sample increases, the dispersion coefficient decreases is shown graphically in the Figure 11:

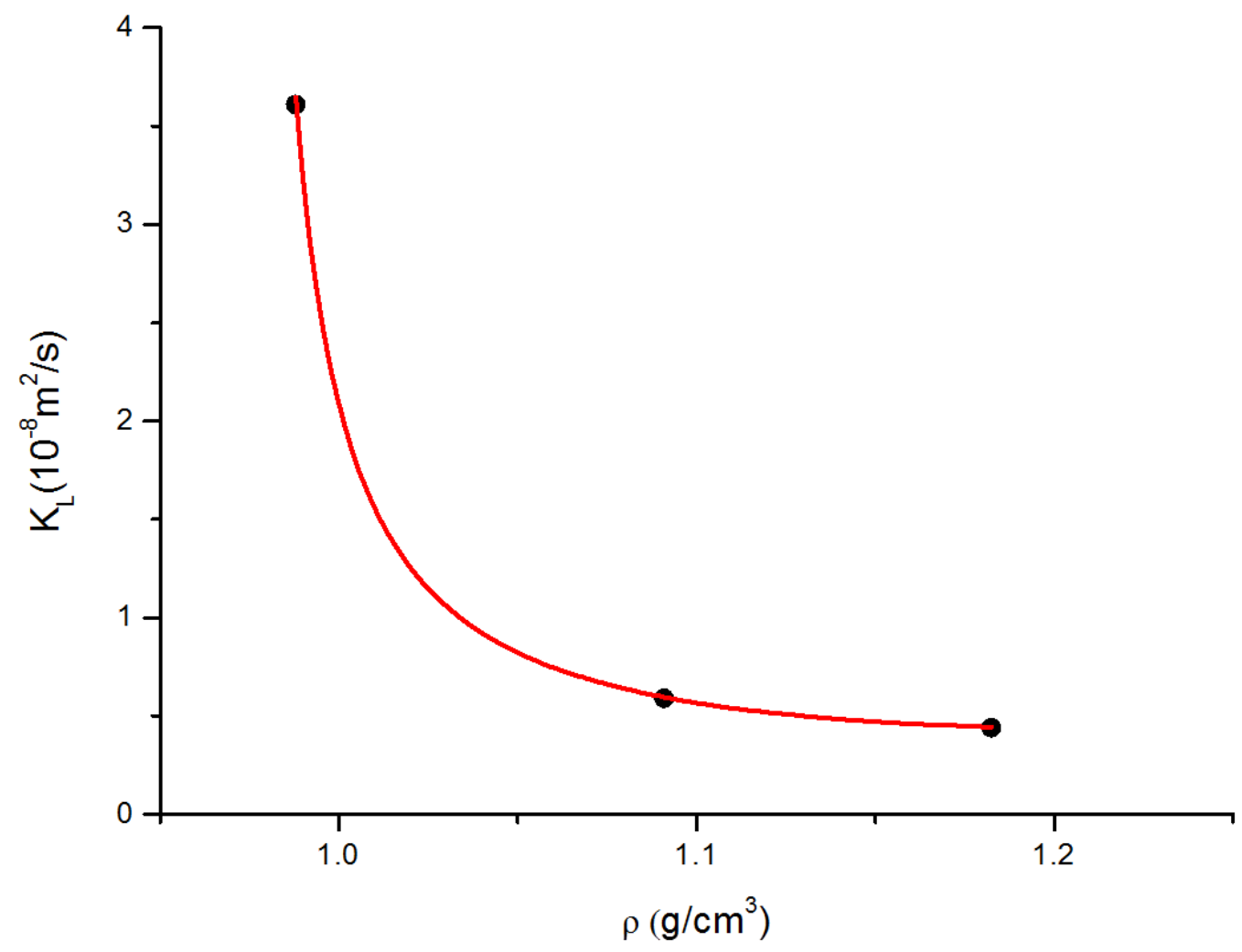

Figure 11: Dispersion Coefficient as a function of connate water density

Albeit the good fit of the data in the graph, the standard error in the fit-line was within $1 \%$ of the average of the experimental data. The graph is mainly for representation and not aimed at describing a model to relate these two properties as there is no data, to the knowledge of this research, found in literature to back up this finding. However, this is a new data in the description of the $\mathrm{CO}_{2}$ dispersion in $\mathrm{CH}_{4}$ in consolidated porous media at conditions relevant to EGR.

The time it will take for the injected $\mathrm{CO}_{2}$ to pass through the core sample will grossly be reduced since the tortuosity is reduced by the inclusion of connate in the core sample given the homogeneous nature of the core sample. This can explain what was observed in the experimental runs with higher density connate water as shown in Figure 12. The pressure drop across the core sample during the run with $10 \%$ wt connate water was considerably higher in comparison with the other concentrations. Due to the high density of $10 \mathrm{wt} \%$ connate water $\left(1.18245 \mathrm{~g} / \mathrm{cm}^{3}\right)$, the capillary forces within the narrower pores in the core sample were overcome and the connate water occupied those pores thereby sealing some of the flow paths within the pore network. This 
reduces the flow channels, significantly, through which the injected $\mathrm{CO}_{2}$ will flow to displace the nascent $\mathrm{CH}_{4}$ which will eventually lead to higher pressure build-up in the core sample as the $\mathrm{CO}_{2}$ transverses the now less tortuous and more constricted core matrix. With this higher pressure drop $(\Delta \mathrm{P})$, a lower permeability is evident according to Darcy relationship between permeability (k) and $\Delta \mathrm{P}$ which states that permeability is inversely proportional to the differential pressure across a core sample as shown in (Eq. 10). The injected $\mathrm{CO}_{2}$ permeability decreased with increase in the density of the connate water.

$$
k=\frac{q \mu L}{A \Delta P}
$$

Where $k$ is the permeability (md), $q$ is flowrate $\left(\mathrm{cm}^{3} / \mathrm{s}\right), \mu$ is fluid viscosity (cp), A is cross sectional area of core $\left(\mathrm{cm}^{2}\right)$, and $\Delta P$ is differential pressure across the core sample (atm).

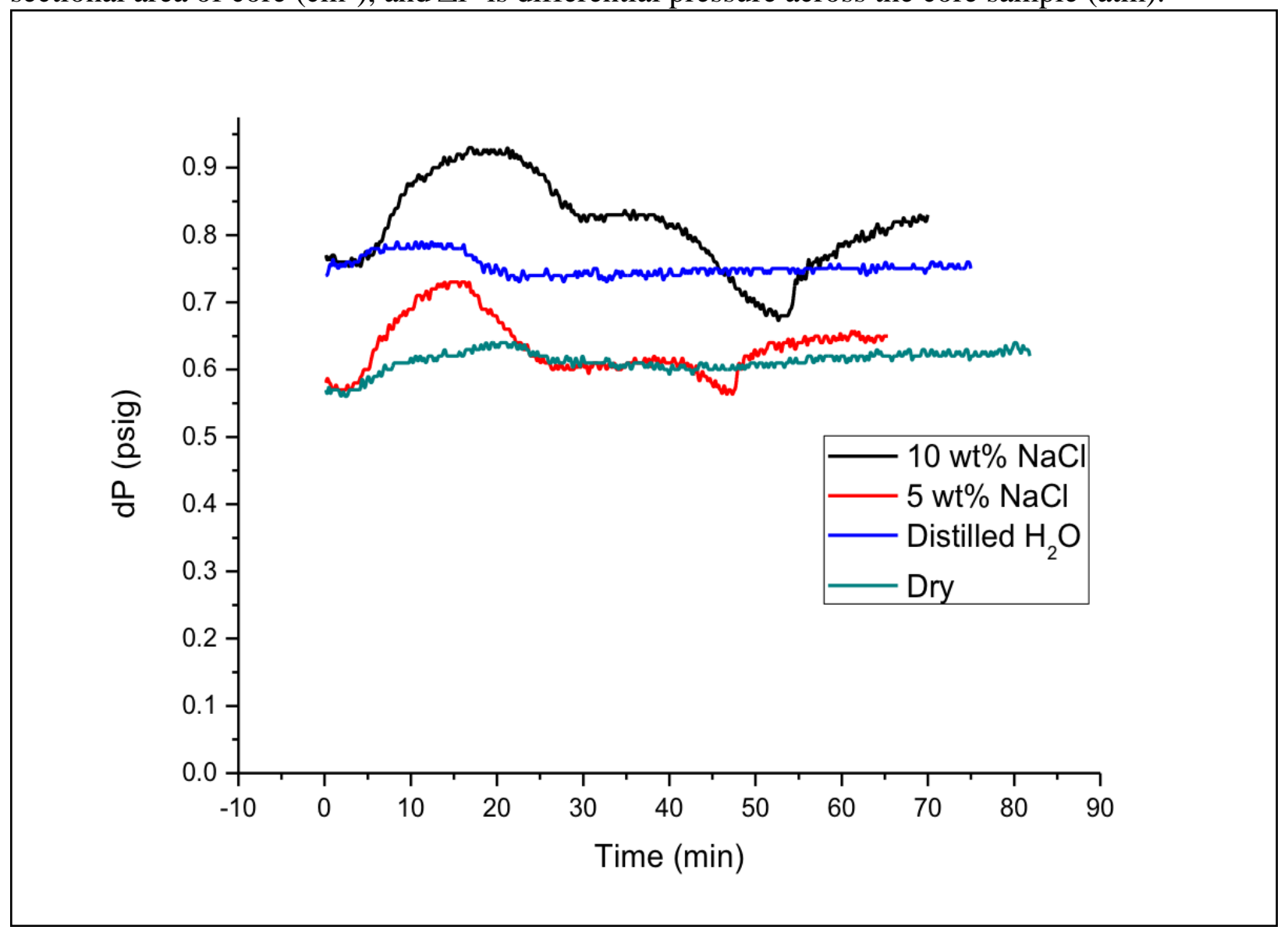

Figure 12: Differential pressure of the experimental runs as a function of time

However, the experimental run with distilled water showed a higher differential pressure, invariably lower permeability, compared to that of the $5 \mathrm{wt} \%$. This can be as result of the reason given by (Abba et al., 2017) that due to the lower density of the distilled water compared to that of the brine, the capillary forces within the pore matrix were not overcome by the density of the distilled water. Therefore, the distilled water did not entirely block or seal off the narrower flow paths, as in the case of the $10 \mathrm{wt} \%$ connate water, of the core sample as suggested by (Honari et al., 2016). Instead, it made it narrower and that decreased the permeability of the core sample 
and this phenomenon also explains the high dispersion coefficient observed with the Distilled water experimental run as shown in Table 4. This clearly shows the influence of connate water salinity on the dispersion coefficient, invariably the mixing of the gases during EGR. For 5wt \% brine, the low $\Delta \mathrm{P}$ and high permeability translated to the sealing off narrower and smaller pore spaces, some of whose capillary forces superseded the density of the brine in question. Therefore, more flow channels were available for flow without impending restrictions as seen in the case of the $10 \mathrm{wt} \%$ brine experiment. Thus, the flow behavior was close to that of the dry run were there was no inclusion of connate water. In higher salinity connate water environment, a lower mixing is expected because of the more homogenous flow paths as discussed earlier. This finding will be vital in the accurate depiction of EGR during simulation studies for field scale applications of the technique.

\section{Conclusion}

The optimum flow conditions for the connate water salinity effect on dispersion coefficient were successfully evaluated through systematic and comparative experimental. These were based on the $\mathrm{CH}_{4}$ recovery and favorable dispersion coefficient of each investigated injection rate. Optimum flow conditions obtained were used to carry out the connate water salinity investigation. From the results, it can be inferred that an increase in the brine density, as a result of increasing its concentration, increased the dispersion coefficient. The density of the connate water plays a significant role in the flow behavior of the injected $\mathrm{CO}_{2}$ in a way that it dictates the flow channels and matrix of the reservoir rock through which the displacement process develops. As seen with the experimental run using the $10 \mathrm{wt} \%$ brine with a density of $1.18245 \mathrm{~g} / \mathrm{cm}^{3}$, the dispersion coefficient was 8 times less than that of the distilled water with density of 0.98796 $\mathrm{g} / \mathrm{cm}^{3}$. This will have a major effect on the contamination of the produced natural gas from the reservoir through EGR. Thus, inclusion of connate water salinity in simulation studies for EGR field application could provide significant understanding of realistic displacement process in sandstones reservoirs.

\section{Acknowledgement}

The authors wish to gratefully acknowledge Petroleum Technology Development Fund (PTDF) Nigeria for the studentship. The support from the Spray Research Group (SRG) will not go unnoticed and also the consultation and input of Petroleum Research Group (PTRG) University of Salford UK.

\section{Nomenclature}

$B_{g} \quad$ Gas formation volume factor, $\mathrm{cm}^{3} / \mathrm{scm}^{3}$

$C \quad \mathrm{CO}_{2}$ mole fraction

$D \quad$ Diffusion coefficient, $\mathrm{m}^{2} / \mathrm{s}$

$d \quad$ Characteristic length scale, $\mathrm{m}$

$G \quad$ Original Gas in place, $\mathrm{cm}^{3}$

$k \quad$ Permeability, md

$K_{L} \quad$ Longitudinal dispersion, $\mathrm{m}^{2} / \mathrm{s}$

$L \quad$ Core sample length, $\mathrm{mm}$

$L_{\text {exp }} \quad$ Experimental length, $\mathrm{m}$ 


$\begin{array}{ll}\mu & \text { Viscosity, } \mathrm{cP} \\ P & \text { Pressure, psig } \\ P_{e} & \text { Peclet number } \\ P_{e m} & \text { Medium Peclet number } \\ Q & \text { Flowrate, } \mathrm{ml} / \mathrm{min} \\ R & \text { Radius of core sample, } \mathrm{mm} \\ S_{w} & \text { Connate water saturation } \\ T & \text { Temperature, }{ }^{\mathrm{O}} \mathrm{C} \\ t & \text { Time, min } \\ t_{D} & \text { Dimensionless time } \\ u & \text { interstitial velocity, } \mathrm{mm}^{2} / \mathrm{s} \\ x & \text { Distance from the upstream of the core face, } \mathrm{m} \\ x_{D} & \text { Dimensionless distance } \\ \phi & \text { Core porosity }\end{array}$

\section{References}

Abba, M.K., Abbas, A.J., Nasr, G.G., 2017. Enhanced Gas Recovery by CO2 Injection and Sequestration: Effect of Connate Water Salinity on Displacement Efficiency. SPE Abu Dhabi Int. Pet. Exhib. Conf. https://doi.org/10.2118/188930-MS

Adepoju, O.O., Lake, L.W., Johns, R.T., Energy, E.M.S., 2013. SPE 166390 Anisotropic Dispersion and Upscaling for Miscible Displacement 421-432.

Al-abri, A., Sidiq, H., Amin, R., 2009. Enhanced Natural Gas and Condensate Recovery by Injection of Pure SCCO2, Pure $\mathrm{CH} 4$ and Their Mixtures : Experimental Investigation. SPE Annu. Tech. Conf. Exhib. New Orleans, Lousiana, USA, 4-7 Oct. 1-13.

Al-Abri, A., Sidiq, H., Amin, R., 2012. Mobility ratio, relative permeability and sweep efficiency of supercritical $\mathrm{CO} 2$ and methane injection to enhance natural gas and condensate recovery: Coreflooding experimentation. J. Nat. Gas Sci. Eng. 9, 166-171. https://doi.org/10.1016/j.jngse.2012.05.011

Al-Abri, A.S., 2011. Enhanced Gas Condensate Recovery by CO 2 Injection.

Allen, R., Nilsen, H.M., Andersen, O., Lie, K.A., 2017. Categorization of Norwegian Continental Shelf Formations in Terms of Geological CO2Storage Potentials. Energy Procedia 114, 4583-4594. https://doi.org/10.1016/j.egypro.2017.03.1579

Bennaceur, K., 2013. CO2Capture and Sequestration. Futur. Energy Improv. Sustain. Clean Options our Planet 583-611. https://doi.org/10.1016/B978-0-08-099424-6.00026-0

Benson, S., Cook, P., Anderson, J., Bachu, S., Nimir, H.B., Basu, B., Bradshaw, J., Deguchi, G., 2005. Underground geological storage. Ipcc 195-276.

Benson, S.M., Cole, D.R., 2008. CO2 sequestration in deep sedimentary formations. Elements 4, 325-331. https://doi.org/10.2113/gselements.4.5.325

Coats, K.H., Smith, B.D., van Genuchten, M.T., Wierenga, P.J., 1964. Dead-End Pore Volume and Dispersion in Porous Media. Soil Sci. Soc. Am. J. 4, 73-84. https://doi.org/10.2118/647-PA

Coats, K.H., Whitson, C.H., Thomas, K., 2009. Modeling Conformance as Dispersion. SPE Reserv. Eval. Eng. 12, 33-47. https://doi.org/10.2118/90390-PA

Ekwere, P.J., 2007. Petrophysics 1049.

Honari, A., Bijeljic, B., Johns, M.L., May, E.F., 2015. Enhanced gas recovery with \{CO2\} 
sequestration: The effect of medium heterogeneity on the dispersion of supercritical CO2CH4. Int. J. Greenh. Gas Control 39, 39-50.

https://doi.org/http://dx.doi.org/10.1016/j.ijggc.2015.04.014

Honari, A., Hughes, T.J., Fridjonsson, E.O., Johns, M.L., May, E.F., 2013. Dispersion of supercritical $\mathrm{CO} 2$ and $\mathrm{CH} 4$ in consolidated porous media for enhanced gas recovery simulations. Int. J. Greenh. Gas Control 19, 234-242.

https://doi.org/10.1016/j.ijggc.2013.08.016

Honari, A., Zecca, M., Vogt, S.J., Iglauer, S., Bijeljic, B., Johns, M.L., May, E.F., 2016. The impact of residual water on $\mathrm{CH} 4-\mathrm{CO} 2$ dispersion in consolidated rock cores. Int. J. Greenh. Gas Control 50, 100-111. https://doi.org/10.1016/j.ijggc.2016.04.004

Hughes, T.J., Honari, A., Graham, B.F., Chauhan, A.S., Johns, M.L., May, E.F., 2012. CO2 sequestration for enhanced gas recovery: New measurements of supercritical $\mathrm{CO} 2-\mathrm{CH} 4$ dispersion in porous media and a review of recent research. Int. J. Greenh. Gas Control 9, 457-468. https://doi.org/10.1016/j.ijggc.2012.05.011

Jha, R.K., Bryant, S.L., Lake, L.W., John, A., 2013. Investigation of Pore-Scale (Local) Mixing. SPE/DOE Symp. Improv. Oil Recover. https://doi.org/10.2118/99782-MS

Kalra, S., Wu, X., 2014. CO2 injection for Enhanced Gas Recovery. SPE West. North Am. Rocky Mt. ... 16-18.

Khan, C., Amin, R., Madden, G., 2013. Carbon dioxide injection for enhanced gas recovery and storage (reservoir simulation). Egypt. J. Pet. 22, 225-240. https://doi.org/10.1016/j.ejpe.2013.06.002

Liu, S., Zhang, Y., Xing, W., Jian, W., Liu, Z., Li, T., Song, Y., 2015. Laboratory experiment of $\mathrm{CO} 2-\mathrm{CH} 4$ displacement and dispersion in sandpacks in enhanced gas recovery. J. Nat. Gas Sci. Eng. 26, 1585-1594. https://doi.org/10.1016/j.jngse.2015.04.021

Mamora, D.D., Seo, J.G., 2002. Enhanced Recovery by Carbon Dioxide Sequestration in Depleted Gas Reservoirs. SPE Annu. Tech. Conf. Exhib. 1-9.

Newberg, M.., Foh, S.., 1988. Measurement of Longitudinal Dispersion Coefficients for Gas Flowing Through Porous Media. SPE 5-9.

Oldenburg, C.M., Benson, S.M., 2002. CO2 Injection for Enhanced Gas Production and Carbon Sequestration. SPE Int. Pet. Conf. Exhib. Mex. https://doi.org/10.2118/74367-MS

Perkins, T.., Johnston, O.., 1963. A Review of Diffusion and Dispersion in Porous Media. Soc. Pet. Eng. J. 3, 70-84. https://doi.org/10.2118/480-PA

Riis, F., Halland, E., 2014. CO2 storage atlas of the Norwegian Continental shelf: Methods used to evaluate capacity and maturity of the $\mathrm{CO} 2$ storage potential. Energy Procedia 63, 52585265. https://doi.org/10.1016/j.egypro.2014.11.557

Sanguinito, S., Goodman, A.L., Sams, J.I., 2018. CO2-SCREEN tool: Application to the oriskany sandstone to estimate prospective CO2storage resource. Int. J. Greenh. Gas Control 75, 180-188. https://doi.org/10.1016/j.ijggc.2018.05.022

Shabani, B., Vilcáez, J., 2017. Prediction of CO2-CH4-H2S-N2gas mixtures solubility in brine using a non-iterative fugacity-activity model relevant to CO2-MEOR. J. Pet. Sci. Eng. 150, 162-179. https://doi.org/10.1016/j.petrol.2016.12.012

Shtepani, E., 2006. CO2 sequestration in depleted gas/condensate reservoirs. Proc. - SPE Annu. Tech. Conf. Exhib.

Sidiq, H., Amin, R., 2009. Mathematical model for calculating the dispersion coefficient of super critical CO2 from the results of laboratory experiments on enhanced gas recovery. J. Nat. Gas Sci. Eng. 1, 177-182. https://doi.org/10.1016/j.jngse.2009.11.001 
Sidiq, H., Amin, R., der Steen, E. Van, Kennaird, T., 2011a. Super critical CO2-methane relative permeability investigation. J. Pet. Sci. Eng. 78, 654-663. https://doi.org/10.1016/j.petrol.2011.08.018

Sidiq, H., Amin, R., der Steen, E. Van, Kennaird, T., 2011b. Super critical CO2-methane relative permeability investigation. J. Pet. Sci. Eng. 78, 654-663. https://doi.org/10.1016/j.petrol.2011.08.018

Sim, S., Turta, A.T., Singhal, A., Hawkins, B.F., 2009. Enhanced Gas Recovery: Effect of Reservoir Heterogeneity on Gas-Gas Displacement. Candian Int. Pet. Conf. 1-14. https://doi.org/10.2118/2009-023

Sim, S., Turta, A.T., Singhal, A.K., Hawkins, B.F., 2009. Enhanced gas recovery: Factors affecting gas-gas displacement efficiency. J. Can. Pet. Technol. 48, 49-55. https://doi.org/10.2118/09-08-49

Sim, S.S.K., Brunelle, P., Canada, Q., Systems, F., Turta, A.T., Singhal, A.K., 2008. SPE 113468 Enhanced Gas Recovery and CO 2 Sequestration by Injection of Exhaust Gases from Combustion of Bitumen. Changes 1-10.

Sminchak, J.R., Babarinde, O., Gupta, N., 2017. Integrated Analysis of Geomechanical Factors for Geologic CO2Storage in the Midwestern United States. Energy Procedia 114, 3267 3272. https://doi.org/10.1016/j.egypro.2017.03.1458

Turta, A.T., Sim, S.S.K., Singhal, A.K., Hawkins, B.F., 2007. Basic Investigations on Enhanced Gas Recovery by Gas-Gas Displacement.

Vilcáez, J., 2015. Numerical modeling and simulation of microbial methanogenesis in geological CO2storage sites. J. Pet. Sci. Eng. 135, 583-595. https://doi.org/10.1016/j.petrol.2015.10.015

Zhang, Y., Liu, S., Song, Y., Zhao, J., Tang, L., Xing, W., Jian, W., Liu, Z., Zhan, Y., 2014. Experimental investigation of $\mathrm{CO} 2-\mathrm{CH} 4$ displacement and dispersion in sand pack for enhanced gas recovery. Energy Procedia 61, 393-397. https://doi.org/10.1016/j.egypro.2014.11.1133

Ziabakhsh-Ganji, Z., Kooi, H., 2012. An Equation of State for thermodynamic equilibrium of gas mixtures and brines to allow simulation of the effects of impurities in subsurface CO2storage. Int. J. Greenh. Gas Control 11, 21-34. https://doi.org/10.1016/j.ijggc.2012.07.025 